\title{
A mid-infrared imaging survey of post-AGB stars
}

\author{
Eric Lagadec $^{1}$, Tijl Verhoelst ${ }^{2}$, Djamel Mékarnia ${ }^{3}$, Olga Suárez ${ }^{3,4}$, \\ Albert A. Zijlstra ${ }^{5}$, Philippe Bendjoya ${ }^{3}$, Ryszard Szczerba ${ }^{6}$, \\ Olivier Chesneau ${ }^{3}$, Hans Van Winckel ${ }^{2}$, Michael J. Barlow ${ }^{7}$, Mikako \\ Matsuura $^{7,8}$, Janet E. Bowey ${ }^{7}$, Silvia Lorenz-Martins ${ }^{9}$, Tim Gledhill ${ }^{10}$ \\ ${ }^{1}$ European Southern Observatory,Garching, Germany \\ email: elagadec@eso.org \\ ${ }^{2}$ Katholieke Universiteit Leuven, Belgium \\ ${ }^{3}$ Laboratoire Fizeau,Nice, France \\ ${ }^{4}$ Instituto de Astrofsica de Andaluca, Granada, Spain \\ ${ }^{5}$ Jodrell Bank Centre for Astrophysics, UK \\ ${ }^{6}$ N. Copernicus Astronomical Center, Torun, Poland \\ ${ }^{7}$ University College London, Gower Street, London \\ ${ }^{8}$ Mullard Space Science Laboratory, University College London, UK \\ ${ }^{9}$ Observatorio do Valongo, Rio de Janeiro, Brazil \\ ${ }^{10}$ Science and Technology Research Institute, University of Hertfordshire
}

\begin{abstract}
Post-AGB stars are key objects for the study of the dramatic morphological changes of low- to intermediate-mass stars on their evolution from the Asymptotic Giant Branch (AGB) towards the planetary nebula stage. There is growing evidences that binary interaction processes may very well have a determining role in the shaping process of many objects, but so far direct evidence is still weak. We aim at a systematic study of the dust distribution around a large sample of post-AGB stars as a probe of the symmetry breaking in the nebulae around these systems. We used imaging in the mid-infrared to study the inner part of these evolved stars to probe direct emission from dusty structures in the core of post-AGB stars in order to better understand their shaping mechanisms. We imaged a sample of 93 evolved stars and nebulae in the mid-infrared using VISIR/VLT, T-Recs/Gemini South and Michelle/Gemini North. We found that all the the proto-planetary nebulae we resolved show a clear departure from spherical symmetry. 59 out of the 93 observed targets appear to be non resolved. The resolved targets can be divided in two categories. The nebulae with a dense central core, that are either bipolar and multipolar. The nebulae with no central core have an elliptical morphology. The dense central torus observed likely host binary systems which triggered fast outflows that shaped the nebulae.
\end{abstract}

Keywords. Stars: AGB and post-AGB, infrared: stars, surveys

\section{Introduction}

Many mid-infrared imaging observations of AGB and PPNe have been made in the past. But the only mid-infrared imaging survey has been made with 3 -m class telescopes (Meixner et al. 1999) and present a lack of angular resolution for the morphological study of the observed objects, a selection bias as they observed known bipolar nebulae and consists of only 17 resolved sources. Some work has been done using 8-m class telescopes, but always focusing on particular individual bright well-known objects.

We observed 86 evolved stars using VISIR at the VLT, 5 using T-Recs on Gemini South and 5 using Michelle on Gemini North. Here we present this mid-infrared N-band imaging survey of a large number of post-AGB stars (Lagadec et al., 2011). We aim at a systematic survey to probe the inner dusty regions of post-AGB stars. 


\section{Target selection and observed morphologies}

Most of the stars observed were selected from the Torun post-AGB stars catalogue (Szczerba et al. 2007). We selected all the post-AGB stars observable in July from the VLT with an IRAS $12 \mu \mathrm{m}$ flux larger than $10 \mathrm{Jy}$ ). In addition to that, we observed the brightest Water Fountains, and AGB stars observable during this period and some objects selected from the previous mid-infrared catalogue by Meixner et al. (1999) and the millimetre observations compiled by Bujarrabal et al. (2001). We also selected star with double chemistry, the unidentified 21-micron features or hints of the presence of an equatorial dusty disc/torus.

We observed 93 targets: 52 PPNe, 10 Water Fountains, 11 AGB stars, 8 RV Tauri stars, 4 PNe, 4 massive evolved stars, 2 R CrB stars, one Be star and one HII region.

According to our measurements, 59 of the 93 objects observed are point sources. Among the extended targets, we resolved a wealth of different structures, such as resolved central cores, dark central lanes, detached shells, S-shaped outflows. If we consider only the PPNe from our sample, we end up with a sample of 52 detected objects. 29 of this objects are not resolved and 6 are marginally resolved. Among the 17 clearly resolved objects, we find 3 ellipticals, 10 bipolars and 4 multipolars. Half of the 10 Water Fountains we observed are resolved. None of the resolved ones are spherical. We observed 4 bipolar Water Fountains, most of them with a dark equatorial lane, and one multipolar.

\section{Discussion}

\subsection{Two kinds of objects: resolved cores and detached shells}

For the largest objects that are clearly resolved, we can notice that the PPNe observed can be divided into two categories (Fig. 1): on one side the objects with a dense central core, in the form of a bright central source, resolved or not, or a dark lane, resolved or not, with most of the emission coming from the poles indicating the presence of

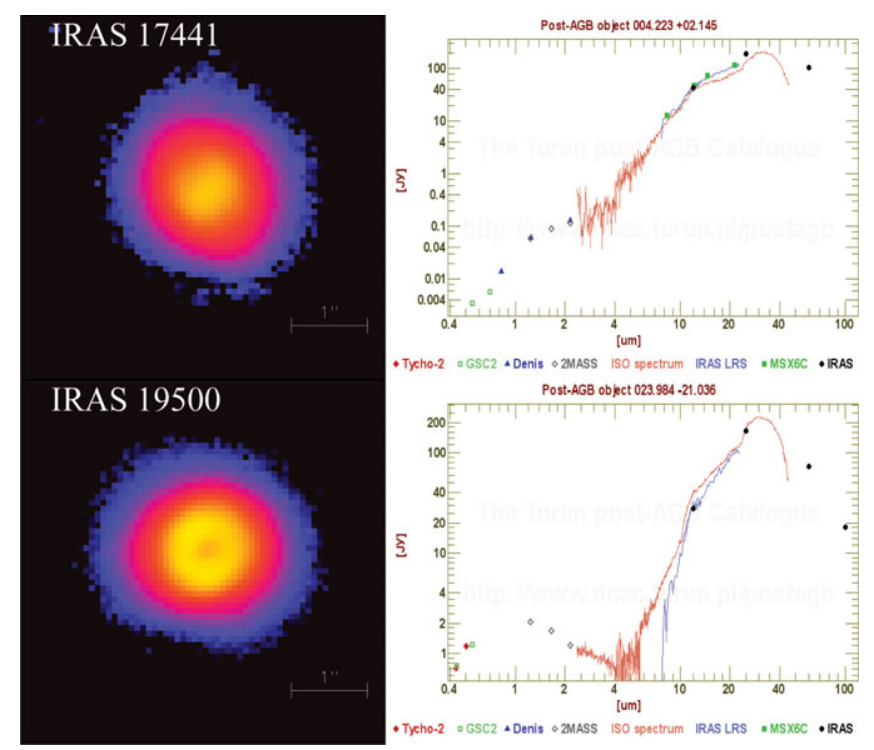

Figure 1. The objects we resolved can be divided in two categories. The targets with a detached shell are elliptical and have a double peak SED. The targets with a central core are bipolar and have a near-infrared excess due to the presence of hot dust close to the star. 
a large amount of dust, making the central regions optically thick even in the midinfrared. On the other side, some objects do not have such a central core, and we can observe either a detached shell or the central star. The objects without a central core all have an elliptical morphology, while the objects with a central core are either bipolar or multipolar. This can be seen in their Spectral Energy Distribution (SED), as the objects with a dense central core or an equatorial dark lane have a rather flat SED in the near-infrared wavelength range, due to the presence of hot dust close to the central star. The SED of the objects with detached shells are characterized by the presence of a clear double-peaked distribution, with a first peak shorter than 1 micron due to the central star, and a second peak due to the cool dust in the shell. The flux is much lower in the near-IR due to the absence of dust close to the central star.

The correlation between the presence of an infrared excess due to hot dust in the core and the bipolar morphology is an indication that the dense cores play a role in the shaping of the nebulae.

\subsection{No spherical PPNe}

We resolved 25 PPNe in our survey. All these nebula show a clear departure from circular symmetry. Some circular shells are resolved in our survey, but only around massive evolved stars. A dramatic change in the the distribution of the circumstellar material is often observed when a star evolves from the AGB phase to the PN phase (Balick \& Frank, 2002). Most AGB stars have a large scale circularly symmetric morphology (Mauron \& Huggins, 2006), while PNe display a variety of morphologies from elliptical to bipolar or multipolar. Parker et al. (2006), from a large optical imaging survey of PNe, found that $\sim 20 \%$ of the PNe are spherical. The shaping of the PNe is thought to occur at the very end of the AGB phase or the beginning of the PPN phase. It is thus surprising that in our sample of 25 resolved PPNe we do not find any circular ones.

The fact that we do not observe any circular PPNe could be a sample selection effect. We selected our targets as bright IRAS $12 \mu \mathrm{m}$ sources. To be a bright emitter at these wavelengths, an object needs to have some dust hot enough $(\sim 300 \mathrm{~K})$, and thus not far from the central star. This is the case for the stars with a central core, which are aspherical, as mentioned before. The spherical PPNe are fainter than the non spherical ones in the mid-infrared, due to the lack of central torus/disc emitting in this wavelength range. At the end of the AGB phase, the envelope of the AGB progenitors of circular PPNe are ejected and rapidly cool down while expanding. There are thus very few spherical PPNe that are bright in the mid-infrared. Furthermore those bright PPNe are compact and thus difficult to spatially resolve. The best way to detect such spherical envelopes is thus at longer wavelengths.

\subsection{Formation of $S$-shaped structures}

For IRAS 17441 a tilt is observed between the orientation of the central dusty torus we resolved and the tips of the observed S-shaped structure. Such a tilt was observed by Volk et al. (2007), and measured to be almost 90 degrees. They suggested that a precession of the dusty torus could explain the observed S-shaped structure of the nebula. They estimated the dynamical age of the envelope, assuming a distance of $1 \mathrm{kpc}$ and an expansion velocity of $100 \mathrm{~km} / \mathrm{s}$, to be $\sim 100 \mathrm{yr}$. According to this, the torus should thus precess with a rate of $\sim 1^{\circ} / \mathrm{yr}$. As our observations were made 4 years after the observations presented by Volk et al. (2007), we should see a tilt of the torus of $4^{\circ}$ between the two observations. The images provided by these authors show that the orientation of the torus they observed is exactly the same as the one we observed. The torus in the core of IRAS 17441 is thus not precessing at such a high rate. 
It thus appears that we can not explain the observed S-shaped structure with a precession of the central tori. This could be due either to the fact that we underestimated the dynamical age of the nebula or that another mechanism is responsible for the S-shaped structure. A more plausible explanation is that the S-shaped structure is not due to the precession of the torus itself, but to precessing outflows inside this torus.

\subsection{Chemistry and morphology}

Amongst the PPNe in our survey, 18 have a known dust chemistry: oxygen, carbonrich or a dual dust chemistry with both carbonaceous and oxygeneous dust grains in their envelopes. For the oxygen-rich sources, we find that 10 out of 11 are bipolar or multipolar, while the remaining one is elliptical. For the carbon-rich sources, we find that 2 are bipolar or multipolar and 2 elliptical. The three objects with a dual dust chemistry are bipolar. This is in agreement with the work by Guzman-Ramirez et al. (2011), which shows a strong correlation between dual dust chemistry and the presence of an equatorial overdensity. The dual dust chemistry could be due either to the formation of PAHs in an oxygen-rich torus after $\mathrm{CO}$ photodissociation, or to the presence of a long-lived O-rich disc formed before the star turned carbon-rich due to the third dredge-up.

In their mid-infrared catalogue, Meixner et al. (1999) used a different morphological classification and found that most of the elliptical source they resolved are O-rich, while the toroidal ones tend to be C-rich. Stanghellini et al. (2007) also studied the correlation between dust composition and morphologies. They determined, from a study of $41 \mathrm{Mag}-$ ellanic Clouds PNe, that all PNe with O-rich dust are bipolar or highly asymmetric. Our study agrees with this last finding, and it seems that O-rich PPNe appears to be bipolar or multipolar. As discussed by De Marco (2009), the low C/O ratio of these bipolar nebulae could be due to the interaction with a binary companion during a common envelope phase, or in the case of single star evolution, result from conversion of carbon to nitrogen. The common phase interaction will lead to the ejection of the envelope earlier than in the single star evolution scenario, leading to a less efficient dredge-up of carbon, and thus a lower C/O ratio (Izzard et al., 2006). The conversion of carbon to nitrogen occurs for massive AGB stars with the hot bottom burning process. It is thus likely that the bipolar PPNe have progenitor with larger masses than the elliptical ones. This is in agreement with the work by Corradi \& Schwartz (1995), who showed that bipolar PNe tend to have a higher progenitor mass. Soker (1998) proposed that this could be explained in the paradigm of binary system progenitors, as primaries that undergo a common envelope phase, and thus become bipolar, tend to have a higher mass.

\section{References}

Balick, B. \& Frank, A. 2002, ARAEAA, 40, 439

Bujarrabal, V., et al. 2001, A\&A, 377, 868

Corradi, R. L. M. \& Schwarz, H. E. 1995, A\&A, 293, 871

de Marco, O. 2009, PASP, 121, 316

Guzman-Ramirez L., Zijlstra, A. A., Níchuimín, R., et al. 2011, MNRAS, 414, 1667

Izzard, R. G., et al. 2006, A\& A, 460, 565

Lagadec, E., Verhoelst, T., Mékarnia, D., et al. 2011, MNRAS, 417, 32

Mauron, N. \& Huggins, P. J. 2006, A\& A, 452, 257

Meixner, M., et al. 1999, ApJS, 122, 221

Parker, Q. A., et al. 2006, MNRAS, 373, 79

Stanghellini, L., et al. 2007, ApJ, 671, 1669

Soker, N. 1998, ApJ, 496, 833

Szczerba R., et al. 2007, A\& A, 469, 799

Volk, K., Kwok, S., \& Hrivnak, B. J. 2007, ApJ, 670, 1137 